\title{
Joule heating in a flat dechirper
}

\author{
Karl Bane* and Gennady Stupakov \\ SLAC National Accelerator Laboratory, Menlo Park, California 94025, USA \\ Erion Gjonaj \\ Technische Universität Darmstadt, 64289 Darmstadt, Germany
}

(Received 14 March 2017; published 30 May 2017)

\begin{abstract}
We perform Joule power loss calculations for a flat dechirper. We consider the configurations of the beam on-axis between the two plates - for chirp control—and for the beam especially close to one platefor use as a fast kicker. Our calculations use a surface impedance approach, one that is valid when corrugation parameters are small compared to aperture (the perturbative parameter regime). We find that most of the wake power lost by the beam is radiated out to the sides of the plates. For the case of the beam passing by a single plate, we derive an analytical expression for the broadband impedance. Our analytical results are also tested by numerical, time-domain simulations. While our theory can be applied to the LCLS-II dechirper with large gaps, for the nominal apertures we are not in the perturbative regime. With input from computer simulations, we estimate the Joule power loss for nominal apertures (assuming bunch charge of $300 \mathrm{pC}$, repetition rate of $100 \mathrm{kHz}$ ) is $21 \mathrm{~W} / \mathrm{m}$ for the case of two plates, and $24 \mathrm{~W} / \mathrm{m}$ for the case of a single plate.
\end{abstract}

DOI: 10.1103/PhysRevAccelBeams.20.054403

\section{INTRODUCTION}

A corrugated structure device—a so-called dechirper [1] -is being proposed for installation after the end of the linac and before the undulator regions of LCLS-II. Such a device has been installed in LCLS-I [2], where it has been used for energy chirp control and as a fast kicker for selfseeding and two color operation of the FEL [3]. Because of the high repetition rate in LCLS-II compared to LCLS-I, Joule heating of the device by the beam's wakefields may now become significant and a cooling system may be required. Thus, it is important to estimate the amount of Joule heating power that is deposited in the corrugated plates.

In previous work, the surface impedance approach to obtaining the impedances and wakes of a short, high energy bunch in a flat dechirper was derived [4-6]. The resulting formulas were shown to be approximate and valid in the perturbative regime, i.e. when $h / a \ll 1$, with $h$ the corrugation depth and $a$ the half aperture of the dechirper. In this regime (assuming also that $h / p \gtrsim 1$, with $p$ the corrugation period) the impedance can be described by a single mode and the longitudinal wake by a damped cosine function [7]. The new RadiaBeam/SLAC dechirper at nominal parameters ( $h=0.5 \mathrm{~mm}$ and $a=0.7 \mathrm{~mm}$, thus $h / a=0.7$ ),

\footnotetext{
*kbane@slac.stanford.edu
}

Published by the American Physical Society under the terms of the Creative Commons Attribution 4.0 International license. Further distribution of this work must maintain attribution to the author(s) and the published article's title, journal citation, and DOI. however, is not in the perturbative regime. In this nonperturbative regime, it has been shown that the impedance consists of more than one mode [8] and the wake begins with a droop [9]. However, even for this regime analytical fitting functions for the short-range wakes have been derived and also verified by numerical simulation [10].

In previous work on the dechirper, the effect of the corrugations alone was considered, and the effect of the resistance of the boundary metal was ignored. For Joule power loss calculations, however, one needs to include both contributions. Also, in most of the previous work on the impedance of a flat dechirper, the case of the beam passing between two corrugated plates was considered. However, when used as a fast kicker, with the beam passing close to one plate, the second plate no longer influences the beam nor needs to be in the problem. Note, however, that analytical fitting formulas for the short-range wakes for this case have also been recently derived [11] and numerically verified [12].

In the present report, by applying the surface impedance approach to both double and single-plate dechirpers, we obtain analytical estimates of the Joule heating power. The surface impedance approach is used where it is not quite applicable $(h / a=0.7$ and is not small), introducing an error. In addition, our estimates ignore the effect of reflections from the side edges of the dechirper plates. We thus expect our results to underestimate the Joule power losses. Finally, we test the accuracy of these calculations, by performing numerical simulations using the timedomain Maxwell equation solver in CST PARTICLE STUDIO (PS) [13], and also (for verification purposes) with the program PBCI [14]. These calculations are themselves 


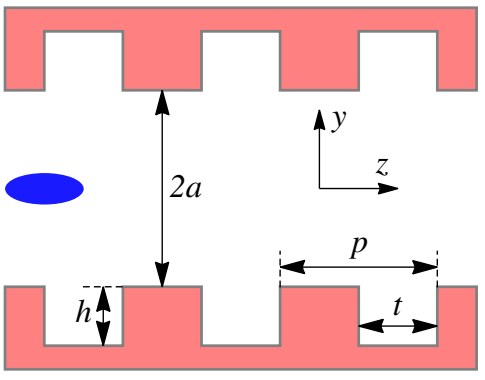

FIG. 1. Three corrugations of a vertical dechirper. A rectangular coordinate system is centered on the symmetry axis of the chamber. The blue ellipse represents an electron beam propagating along the $z$ axis.

quite challenging, since a fine mesh is needed to resolve the short bunch, and runs need to be performed over a large mesh domain for a relatively long time.

The RadiaBeam/SLAC dechirper that is installed in LCLS-I consists of two modules. Each module has two corrugated plates, with the beam passing between them (see Fig. 1). Two modules were chosen in order to partially cancel the unavoidable quadrupole wakefield that is induced by the beam; one has plates parallel to the $x-z$ axis (horizontal-longitudinal) plane and is adjustable vertically (the "vertical dechirper"), and the complementary one is adjustable horizontally (the "horizontal dechirper"). For the new LCLS-II dechirper, the corrugation parameters will be the same: period $p=0.5 \mathrm{~mm}$, (longitudinal) gap $t=0.25 \mathrm{~mm}$, depth $h=0.5 \mathrm{~mm}$; nominally the gap $g=2 a=1.4 \mathrm{~mm}$. The plate length $L=1.5 \mathrm{~m}$ and width $w=12 \mathrm{~mm}$. Our calculations here consider: (i) a vertical two-plate dechirper, with the beam on the symmetry axis (such as is shown in Fig. 1); and (ii) only the top plate, with

TABLE I. Selected beam and machine properties for LCLS-II used in example calculations. This is the high charge option with its maximum repetition rate. The charge distribution is assumed to be uniform with peak current $I=1.5 \mathrm{kA}$. The dechirper properties are those of the RadiaBeam/LSLAC dechirper, which consists of two modules, each with corrugated plates of length $L=2 \mathrm{~m}$. The plates are made of aluminum; we take conductivity $\sigma_{c}=3.2 \times 10^{17} / \mathrm{s}$.

\begin{tabular}{lcc}
\hline \hline Parameter name & Value & Unit \\
\hline Beam energy, $E$ & 6 & $\mathrm{GeV}$ \\
Charge per bunch, $Q$ & 300 & $\mathrm{pC}$ \\
Full bunch length, $\ell$ & 60 & $\mu \mathrm{m}$ \\
Repetition rate, $f_{\text {rep }}$ & 100 & $\mathrm{kHz}$ \\
Dechirper properties: & & \\
Period, $p$ & 0.5 & $\mathrm{~mm}$ \\
Longitudinal gap, $t$ & 0.25 & $\mathrm{~mm}$ \\
Full depth, $h$ & 0.5 & $\mathrm{~mm}$ \\
Nominal half aperture, $a$ & 0.7 & $\mathrm{~mm}$ \\
Plate width, $w$ & 12 & $\mathrm{~mm}$ \\
Plate length, $L$ & 2 & $\mathrm{~m}$ \\
\hline \hline
\end{tabular}

the beam just below it. The dechirper parameters and typical beam and machine properties used in calculations here are given in Table I.

The equations in this report are given in cgs units. To convert an impedance or wake into MKS units, one multiplies the cgs result by $Z_{0} c /(4 \pi)$, with $Z_{0}=377 \Omega$.

\section{JOULE HEATING ESTIMATES}

In a round corrugated structure of a finite length (a dechirper), the wakefield energy loss experienced by a relativistic beam of charged particles is partly absorbed in the walls as Joule heating and partly generates a THz pulse that leaves the structure just behind the driving particle. In the flat geometry of dechirpers like those that have actually been built—like the RadiaBeam/LCLS dechirper-some of that energy can also escape through the aperture to the side. The energy per unit length lost by the beam to the wake is then given by the sum

$$
u_{w}=u_{h}+\left(u_{\mathrm{rad}}\right)_{z}+\left(u_{\mathrm{rad}}\right)_{x},
$$

with $u_{h}$ the energy generating Joule heating in the metal walls, $\left(u_{\mathrm{rad}}\right)_{z}$ the energy in the THz pulse that leaves the end of the structure following the driving particle, and $\left(u_{\mathrm{rad}}\right)_{x}$ the energy radiating out the sides of the structure.

A particle of charge $Q$ moves at the speed of light $c$ on the axis of a structure. The Joule energy loss into the walls per unit length is given by

$$
u_{h}=\frac{1}{c} \int_{\mathcal{B}} \mathbf{S}(\mathbf{r}, z) \cdot d \mathbf{A},
$$

with $S$ the Poynting vector, $d \mathbf{A}$ the incremental surface area vector (into the wall is positive), and $\mathcal{B}$ represents the metallic boundary. The calculation is performed at time $t=0$ when the particle is at $z=0$, and the transverse coordinate is $\mathbf{r}$. The particle is assumed to be moving to the left; the fields are zero ahead of the particle (for $z<0$ ).

Let us begin by sketching how we would solve the case of a dechirper with round geometry. The walls are located at radius $r=a$, and the Poynting vector at the walls is given by $S=-\left(\frac{c}{4 \pi}\right) E_{z} H_{\phi}$, with $E_{z}(r, z)$ the longitudinal component of the electric field and $H_{\phi}(r, z)$ the azimuthal component of the magnetic field. The Joule energy loss into the walls becomes

$$
u_{h}=\frac{a}{2} \int_{-\infty}^{\infty} d z E_{z}(a, z) H_{\phi}(a, z) .
$$

The fields can be written in terms of their Fourier transforms; for example, for the electric field

$$
\begin{aligned}
\tilde{E}_{z}(\omega) & =\frac{1}{c} \int_{0}^{\infty} d z E_{z}(z) e^{i \omega z / c}, \\
E_{z}(z) & =\frac{1}{2 \pi} \int_{-\infty}^{\infty} d \omega \tilde{E}_{z}(\omega) e^{-i \omega z / c},
\end{aligned}
$$


where $\omega$ is the frequency and a tilde indicates the Fourier transform of a field (and similarly for $H_{\phi}$ ). Substituting into Eq. (3) and changing the order of integrations we obtain

$u_{h}=\frac{a}{8 \pi^{2}} \int_{-\infty}^{\infty} d \omega \tilde{E}_{z}(\omega) \int_{-\infty}^{\infty} d \omega^{\prime} \tilde{H}_{\phi}\left(\omega^{\prime}\right) \int_{-\infty}^{\infty} d z e^{-i\left(\omega+\omega^{\prime}\right) z / c}$.

The last integral on the right equals $2 \pi c \delta\left(\omega+\omega^{\prime}\right)$. Thus, we obtain

$u_{h}=\frac{c a}{4 \pi} \int_{-\infty}^{\infty} d \omega \tilde{E}_{z}(\omega) \tilde{H}_{\phi}(-\omega)=\frac{c a}{4 \pi} \int_{-\infty}^{\infty} d \omega \tilde{E}_{z}(\omega) \tilde{H}_{\phi}(\omega)^{*}$,

where in the last integral $*$ indicates the complex conjugate of a function. To obtain the last integral we used the relation $\tilde{H}_{\phi}(-\omega)=\tilde{H}_{\phi}(\omega)^{*}$; such a relation holds for the fields in the frequency domain, since the same fields in the time domain must be real quantities.

On the metallic surface we have the relation

$$
\tilde{E}_{z}(\omega)=\zeta_{z}(\omega) \tilde{H}_{\phi}(\omega),
$$

with $\zeta_{z}(\omega)$ the surface impedance of the structure walls, which includes both the contributions of the corrugations and the wall resistance (the subscript $z$ indicates that the surface currents move in the longitudinal direction). Substituting into Eq. (6), and noting the symmetry of the integrand, we finally obtain

$$
u_{h}=\frac{c a}{2 \pi} \int_{0}^{\infty} d \omega \operatorname{Re}\left[\zeta_{z}(\omega)\right]\left|\tilde{H}_{\phi}(a, \omega)\right|^{2} .
$$

If we write the Joule loss as $u_{h}=\int_{-\infty}^{\infty} \frac{d \tilde{u}}{d \omega} d \omega$, then we can define an effective Joule heating impedance $Z_{h}(\omega)$, which has a real part defined as

$$
\operatorname{Re}\left[Z_{h}(\omega)\right]=\frac{\pi}{Q^{2}} \frac{d \tilde{u}}{d \omega}(\omega) .
$$

In this round example it turns out that all the wake losses end up in the walls, since they have nowhere else to go. It is easy to see that $Z_{h}(\omega)=Z(\omega)$, with $Z(\omega)$ the normally defined impedance of the corrugated structure, given by $Z(\omega)=-\tilde{E}_{z} / Q$. For a bunch of particles, the Joule heating energy is given by

$$
u_{h \lambda}=\frac{1}{\pi} \int_{0}^{\infty} d \omega|\tilde{I}(\omega)|^{2} \operatorname{Re}\left[Z_{h}(\omega)\right],
$$

with $\tilde{I}(\omega)$ the Fourier transform of the current, $I(z)=Q c \lambda(z)$, and $\lambda(z)$ the longitudinal bunch distribution. However, in following energy and power calculations we will let $|\tilde{I}(\omega)|^{2} \rightarrow 1$, since for the small bunch lengths considered and the frequency reach of the dechirper impedance, this approximation is good.

The Joule power loss is simply given by $P=u_{h} f_{\text {rep }}$, with $f_{\text {rep }}$ the bunch repetition rate. In the round case, since all the beam energy loss becomes Joule heating, we obtain $u_{h}=2 Q^{2} / a^{2}$. Our Joule energy loss calculations are perturbative calculations. If, however, the corrugation parameter ratio $(h / a)$ is not small, then we are not in the perturbative regime and there will be wake droop. In such a case, to better estimate the Joule power loss we take

$$
P=f_{\text {rep }} u_{h}\left[\frac{\varkappa\left(\sigma_{z}\right)}{\varkappa(0)}\right],
$$

with $\varkappa\left(\sigma_{z}\right)$ the loss factor of a Gaussian bunch of length $\sigma_{z}$. The point charge loss factor $\varkappa(0)=2 / a^{2}$ (with $a$ the pipe radius) in the round case, and $\varkappa(0)=\pi^{2} /\left(8 a^{2}\right)$ (with $a$ the half aperture) in the flat case. The loss factor $\varkappa\left(\sigma_{z}\right)$ includes the effect of the wake droop.

\section{FLAT GEOMETRY}

In flat geometry energy can also radiate out the sides. To obtain our Joule heating estimate we perform a simple approximate calculation. End on, a vertical dechirper looks like what is shown in the sketch of Fig. 2(a). The vertical gap is $2 a$ and the width of the corrugated plates is $w$. For our calculations we let the width of the plates become infinite, and to account for the finite width as in the real case, we perform Joule loss calculations over the boundary only over a region of width $\pm w / 2$ from the beam path [see Fig. 2(b); the distance between the dashed lines is meant to be $w$ ]. Our calculation is a perturbation calculation that is only accurate when the corrugation parameters are small compared to the aperture $2 a$. Note that the dechirper parameters of Table I are not in the perturbative regime. One contributor to inaccuracy is that we assume that the structure is infinitely long, which does not allow for part of the wake loss to contribute to the generation of a THz pulse in the $z$ direction. However, this contribution can be shown to be small for the parameters of Table I.

Let us consider a vertical dechirper with plate walls of width $w$ located at $y= \pm a$ with respect to the axis. The Poynting vector at the walls

$$
S=\left(\frac{c}{4 \pi}\right)\left(E_{x} H_{z}-E_{z} H_{x}\right) .
$$

Note that in this case, in addition to a surface current in the $z$ direction, corresponding to the surface impedance $\zeta_{z}$, there is a surface impedance in the $x$ direction, corresponding to surface impedance $\zeta_{x}$. The surface impedance in the $z$ direction is given by the sum of the resistive wall and the corrugation impedance contributions, $\zeta_{z}=\zeta_{r w}+\zeta_{\text {corr }}$, with $[4,15]$, 


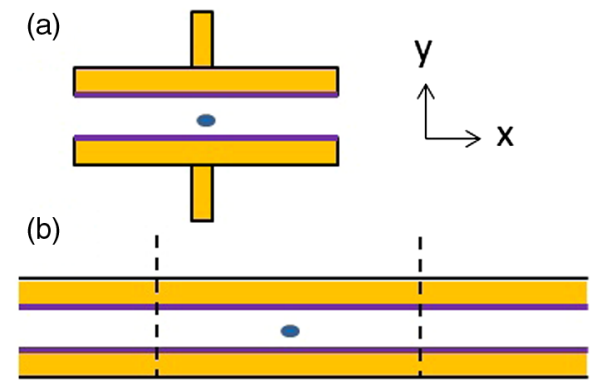

FIG. 2. Sketch of a vertical set of dechirper jaws seen end on: (a) in the real geometry the jaws have a finite width $w$; (b) in the model used in the calculation, the width is infinite, but the loss integration is performed only over a width $w$ (represented by the distance between the dashed lines). The purple lines represent the corrugated surfaces; the blue ellipses, the exciting particle's transverse location.

$\zeta_{r w}(\omega)=\left(\frac{\omega}{8 \pi \sigma_{c}}\right)^{1 / 2}(1-i), \quad \zeta_{\mathrm{corr}}(\omega)=-i \frac{h \omega}{2 c}$,

and $\sigma_{c}$ is the conductivity of the metal walls. In the $x$ direction, we take the surface impedance to be $\zeta_{x}=\zeta_{r w}$, since the horizontal surface currents are not impeded by the corrugations. Note that an anisotropic surface impedance was not used before for modeling the impedance of the corrugated structure; this form, however, is important in our application here. Note also that with only corrugations and no wall resistance, the Joule heating is zero.

The Joule wall energy loss per unit length becomes

$$
\begin{aligned}
u_{h}= & \frac{1}{2 \pi} \int_{-w / 2}^{w / 2} d x \int_{-\infty}^{\infty} d z\left[E_{z}(x, a, z) H_{x}(x, a, z)\right. \\
& \left.+E_{x}(x, a, z) H_{z}(x, a, z)\right]
\end{aligned}
$$

(an overall factor of 2 is added because there are two plates). There are two contributions. Let us take $u_{h}=u_{h z}+u_{h x}$, with $u_{h z}$ the part that depends on $E_{z} H_{x}$ at the walls; $u_{h x}$ the part that depends on $E_{x} H_{z}$ at the walls. Following a calculation similar to that for the round case above, we can rewrite the equation for $u_{z}$ in the frequency domain as

$u_{h z}=\frac{c}{4 \pi^{2}} \int_{-w / 2}^{w / 2} d x \int_{-\infty}^{\infty} d \omega \operatorname{Re}[\zeta(\omega)]\left|\tilde{H}_{x}(x, a, \omega)\right|^{2}$.

To perform the calculation, we follow the procedure described in Ref. [5], which explicitly gives the fields and wakefields in structures with flat geometry for which the effect at the boundaries can be approximated by a surface impedance. First the frequency representation of the fields are Fourier transformed in $x$ as

$$
\begin{aligned}
& \hat{H}_{x}(q, y, \omega)=\int_{-\infty}^{\infty} d x \tilde{H}_{x}(x, y, \omega) e^{i q x}, \\
& \tilde{H}_{x}(x, y, \omega)=\frac{1}{2 \pi} \int_{-\infty}^{\infty} d q \hat{H}_{x}(q, y, \omega) e^{-i q x} .
\end{aligned}
$$

Substituting into Eq. (15), changing the order of integration, and noting the symmetry of the integrand with respect to $\omega$, we find that

$$
\begin{aligned}
u_{h z}= & \frac{c w}{8 \pi^{4}} \int_{0}^{\infty} d \omega \operatorname{Re}\left[\zeta_{z}(\omega)\right] \int_{-\infty}^{\infty} d q \hat{H}_{x}(q, a, \omega) \\
& \times \int_{-\infty}^{\infty} d q^{\prime} \hat{H}_{x}^{*}\left(q^{\prime}, a, \omega\right) \operatorname{sinc}\left[\frac{w\left(q-q^{\prime}\right)}{2}\right] .
\end{aligned}
$$

It is this triple integral that we solve numerically to estimate the fraction of wakefield losses that end up as Joule heating in the walls. Note that for the special case width $w \rightarrow \infty$, the equation simplifies to

$u_{h z}=\frac{c}{2 \pi^{3}} \int_{0}^{\infty} d \omega \operatorname{Re}\left[\zeta_{z}(\omega)\right] \int_{0}^{\infty} d q\left|\hat{H}_{x}(q, a, \omega)\right|^{2}$

(we have used the fact that the integrand is symmetric with respect to $q$ ).

Performing a similar calculation for $u_{x}$, we obtain, for finite $w$,

$$
\begin{aligned}
u_{h x}= & \frac{c w}{8 \pi^{4}} \int_{0}^{\infty} d \omega \operatorname{Re}\left[\zeta_{x}(\omega)\right] \int_{-\infty}^{\infty} d q \hat{H}_{z}(q, a, \omega) \\
& \times \int_{-\infty}^{\infty} d q^{\prime} \hat{H}_{z}^{*}\left(q^{\prime}, a, \omega\right) \operatorname{sinc}\left[\frac{w\left(q-q^{\prime}\right)}{2}\right],
\end{aligned}
$$

and for infinite $w$

$u_{h x}=\frac{c}{2 \pi^{3}} \int_{0}^{\infty} d \omega \operatorname{Re}\left[\zeta_{x}(\omega)\right] \int_{0}^{\infty} d q\left|\hat{H}_{z}(q, a, \omega)\right|^{2}$.

The general form of the impedances for a flat structure, one that can be described using the surface impedance concept, is developed in Refs. [5,6]. A conclusion of this work was that the final results-the impedances-were approximate and valid, provided that the frequency $k=\omega / c \gg 1 / a$-which is satisfied-and that $|\zeta| \ll 1$ (or $h / a \ll 1$ ), which is not for our nominal corrugation parameters (see Table I). The expressions for $\hat{H}_{x}(q, a, \omega)$, $\hat{H}_{z}(q, a, \omega)$, that we need here were not given in the earlier reports. Following the correct derivation of the fields, we obtained the general form of $\hat{H}_{x}(q, a, \omega)$ and $\hat{H}_{z}(q, a, \omega)$ as functions of beam offset (not shown). For the special case of the beam on axis, the fields on the boundary at $y=a$ are 


$$
\begin{aligned}
& \hat{H}_{x}=-\frac{4 \pi Q}{c} \frac{i k q \cosh (a q)}{\zeta_{z}(k-q)(k+q) \sinh (2 a q)+2 i k q\left[\zeta_{z} \zeta_{x} \sinh ^{2}(a q)+\cosh ^{2}(a q)\right]}, \\
& \hat{H}_{z}=-\frac{4 \pi Q}{c} \frac{\zeta_{z} k q \sinh (a q)}{\zeta_{z}(k-q)(k+q) \sinh (2 a q)+2 i k q\left[\zeta_{z} \zeta_{x} \sinh ^{2}(a q)+\cosh ^{2}(a q)\right]},
\end{aligned}
$$

with $k=\omega / c$.

In the case of flat geometry, with corrugated plate width $w \rightarrow \infty$, the total Joule energy loss on the walls, $u_{z}+u_{x}$, must equal a point charge beam's energy loss on the axis, $u_{w}$. In this case the point charge loss factor

$$
\kappa(0)=\frac{u_{w}}{Q^{2}}=-\frac{1}{\pi Q} \int_{0}^{\infty} d \omega \operatorname{Re}\left[\tilde{E}_{z}(\omega)\right]=-\frac{1}{\pi^{2} Q} \int_{0}^{\infty} d \omega \int_{0}^{\infty} d q \operatorname{Re}\left[\hat{E}_{z}(\omega, q)\right],
$$

with the on-axis electric field

$$
\hat{E}_{z}=-\frac{4 \pi Q}{c} \frac{i k q \zeta_{z}}{\zeta_{z}(k-q)(k+q) \sinh (2 a q)+2 i k q\left[\zeta_{z} \zeta_{x} \sinh ^{2}(a q)+\cosh ^{2}(a q)\right]}
$$

The impedance is given by

$$
Z(\omega)=-\frac{\tilde{E}_{z}(\omega)}{Q}=-\frac{1}{2 \pi Q} \int_{-\infty}^{\infty} d q \hat{E}_{z}(q, \omega)
$$

Substituting from Eq. (23) and integrating numerically we obtain the impedance. The real part, for this case $(a=0.7 \mathrm{~mm}$ and $w \rightarrow \infty)$, is shown in Fig. 3. We see that the impedance is a highly spiked function, with the peak location at $k a \approx \sqrt{2 a / h} \approx 1.673$ [7]. Note that a different, earlier perturbation analysis, one that ignored wall resistance, gave essentially the same result except that the spike reached to infinity [7]. This implies that the shortrange wake is essentially independent of the boundary conductivity.

The Joule heating energy has two components, $u_{h z}$ and $u_{h x}$, corresponding to contributions from $\zeta_{z}$ and $\zeta_{x}$,

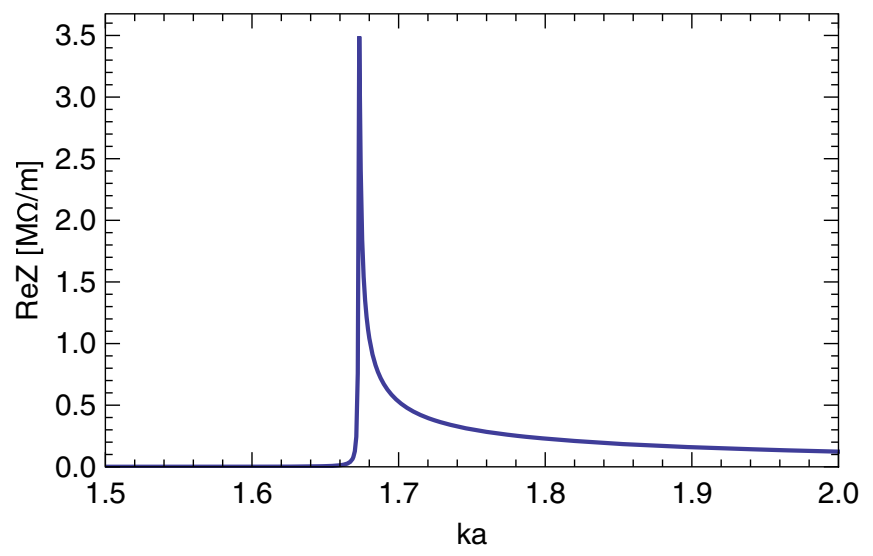

FIG. 3. The real part of the longitudinal impedance for the dechirper with plate width $w \rightarrow \infty$. This plot, in different units, and with the spike reaching to infinity, can also be found in Ref. [7]. respectively. Numerically solving Eqs. (18) and (20) for the infinitely wide plates, we find that $u_{h z}=0.8 u_{w}$, $u_{h x}=0.2 u_{w}$, and, to good accuracy, $u_{h} \equiv u_{h z}+u_{h x}=$ $u_{w}=Q^{2} \pi^{2} /\left(8 a^{2}\right)$. This is what we expect: for plates of infinite width, the sum of the two Joule energy contributions should equal the energy loss of the on-axis point charge beam.

Performing the numerical integrals for finite plate width $w=12 \mathrm{~mm}$, we obtain $u_{h}=u_{h z}+u_{h x}$ using Eqs. (17) and (19). For $a=0.7 \mathrm{~mm}$, we find that the total Joule loss is a small part of the beam energy loss, $u_{h}=0.03 u_{w}$ (with $u_{h z}=0.91 u_{h}$ ). Then using Eq. (9) we obtain the real part of the Joule heating impedances, $Z_{h z}$ and $Z_{h x}$. In Fig. 4 we plot the Joule impedance sum, $\operatorname{Re}\left(Z_{h}\right)=$ $\operatorname{Re}\left(Z_{h z}\right)+\operatorname{Re}\left(Z_{h x}\right)$. Note that on the scale of the plot, $\operatorname{Re}\left(Z_{h}\right) \approx \operatorname{Re}\left(Z_{h z}\right)$ and $\operatorname{Re}\left(Z_{h x}\right) \approx 0$. As a spot check on sensitivity to conductivity, we reduced $\sigma_{c}$ by a factor of 4 ,

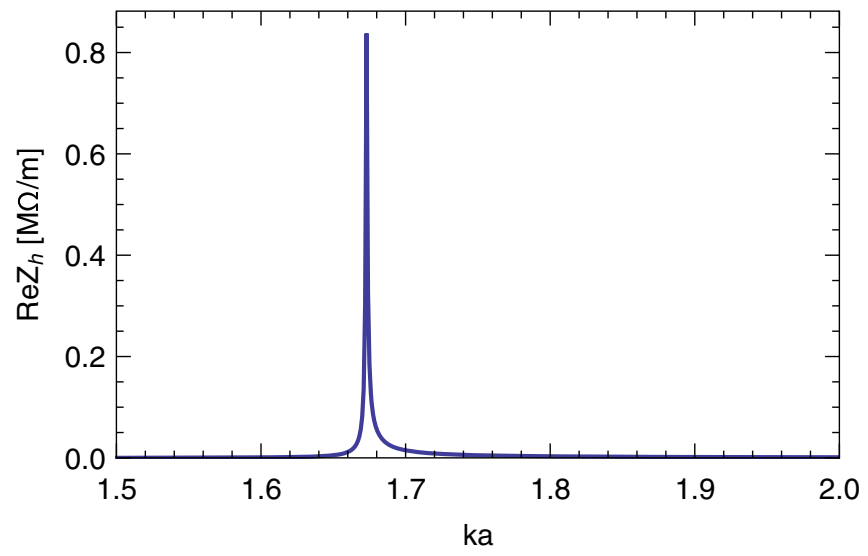

FIG. 4. The real part of the Joule heating impedance $\operatorname{Re}\left(Z_{h}\right)=$ $\operatorname{Re}\left(Z_{h z}\right)+\operatorname{Re}\left(Z_{h x}\right)$ for the beam on axis in the dechirper with half aperture $a=0.7 \mathrm{~mm}$ and plate width $w=12 \mathrm{~mm}$. 


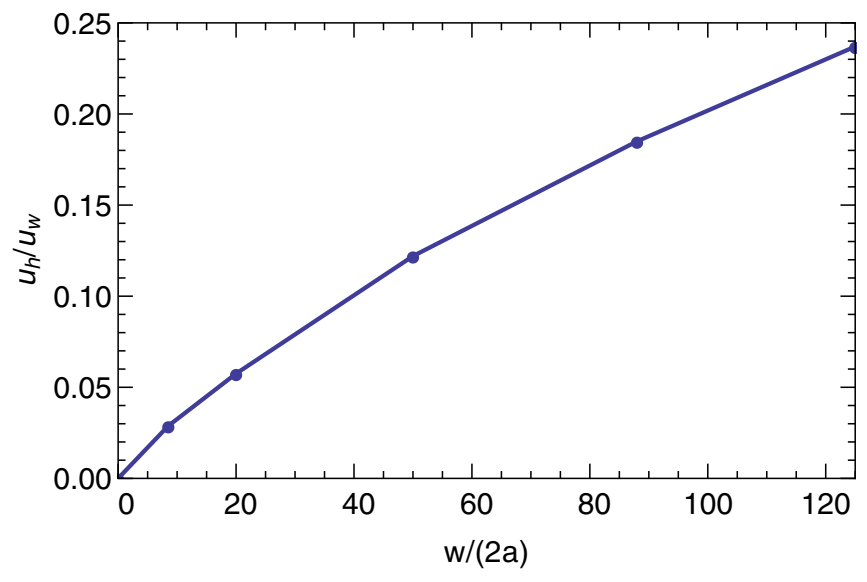

FIG. 5. Numerically calculated ratio of Joule loss into metal to wake loss of a point charge beam, $u_{h} / u_{w}=\left(u_{h z}+u_{h x}\right) / u_{w}$ as a function of $w /(2 a)$ (plotting symbols). The wake loss for a point particle is $u_{w}=Q^{2} \pi^{2} /\left(8 a^{2}\right)$.

repeated the calculation, and found that $\left(u_{h} / u_{w}\right)$ increased by $35 \%$.

We repeat the numerical calculation for several values of plate width $w$. In Fig. 5 we plot the Joule energy loss vs plate width, normalized to the point charge loss of the beam, $u_{w}=Q^{2} \pi^{2} /\left(8 a^{2}\right)$. We see that, even for $w \sim 100 a$, only a small part of the beam energy loss ends up as Joule heating. For our final estimate of Joule power loss of a uniform beam of full length $\ell=60 \mu \mathrm{m}$ we need to use the short-range, point charge wake of a beam on the axis of a flat dechirper [10],

$$
w_{z}(s)=\frac{\pi^{2}}{4 a^{2}} e^{-\sqrt{s / s_{0}}}
$$

with $s_{0}=9 a^{2} t /\left[8 \pi \alpha(t / p)^{2} p^{2}\right]$ and $\alpha(x) \approx 1-0.465 \sqrt{x}-$ $0.070 x$. For the parameters of Table I, the scale factor $s_{0}=434 \mu \mathrm{m}$. For a bunch with uniform distribution of full length $\ell$, the loss factor is given by

$$
\varkappa=\frac{1}{\ell} \int_{0}^{\ell} d s\left(1-\frac{s}{\ell}\right) w_{z}(s)
$$

Here $\varkappa=19 \mathrm{kV} /(\mathrm{pC} * \mathrm{~m})$, and the loss compared to a point charge beam is $\varkappa / \varkappa(0)=0.82$.

For the beam parameters of Table I the power lost by the beam is $P_{w}=Q^{2} \varkappa f_{\text {rep }}=170 \mathrm{~W} / \mathrm{m}$. Thus our analytical estimate of the Joule losses for the $12 \mathrm{~mm}$-wide dechirper plates is the fraction $\left(u_{h} / u_{w}\right)=0.03$ of this, or $P_{h}=5 \mathrm{~W} / \mathrm{m}$.

\section{BEAM NEAR ONE PLATE}

There is interest in streaking the beam by inducing the transverse wakes of the dechirper, by passing the beam close to one jaw. With the beam a distance from the near wall of $b \sim 0.25 \mathrm{~mm}$ and from the far wall by $\gtrsim 5 \mathrm{~mm}$, the second wall will no longer affect the results. The physics will be quite different than before: with two plates the impedance has a narrow resonance whose frequency depends on the plate separation $2 a$; in the single-plate case this parameter no longer exists. We present more details of this case, since the analysis of it is a relatively new topic. Note that in [11] expressions for the wakes for a short beam passing by a single plate of a dechirper are obtained, and that these expressions are numerically verified in [12].

For the Joule heating calculation we start with the equations for the magnetic fields on the wall, for a beam offset by $y$ from the axis of a two-plate dechirper (equations not shown here). We let $y=a-b$, and then let $a \rightarrow \infty$, to obtain the fields on the walls of one plate due to a bunch passing by at distance $b$. The fields on the wall (at $y=b$ ) are given by

$\hat{H}_{x}(q, b, \omega)=-\frac{4 \pi Q}{c} \frac{k|q| e^{-b|q|}}{k|q|\left(1+\zeta_{z} \zeta_{x}\right)+i \zeta_{z}\left(q^{2}-k^{2}\right)}$,

$\hat{H}_{z}(q, b, \omega)=-i \zeta_{z} \hat{H}_{x}(q, b, \omega)$.

Meanwhile the electric field at the particle location (here, at $y=0$ ) is

$$
\hat{E}_{z}(q, 0, \omega)=-\frac{4 \pi Q}{c} \frac{\zeta_{z} k|q| e^{-2 b|q|}}{k|q|\left(1+\zeta_{z} \zeta_{x}\right)+i \zeta_{z}\left(q^{2}-k^{2}\right)} .
$$

First, note that while the point charge energy loss per length of the two-plate system was $u_{w}=Q^{2} \pi^{2} /\left(8 a^{2}\right)$, with $a$ the half gap, for the single plate it is only $u_{w}=Q^{2} /\left(2 b^{2}\right)$, with $b$ the distance between the beam's path and the plate. In Fig. 6 we plot $\operatorname{Re}(Z)$, where $Z=-\tilde{E}_{z} / Q$ (in blue). Instead of the narrow spike of the two-plate case, we now find a relatively broad peak.

Actually, Fig. 6 can be obtained analytically. If we let the resistive terms of both $\zeta_{z}$ and $\zeta_{x}$ be zero, the integral over $q$ that needs to be performed to obtain the impedance

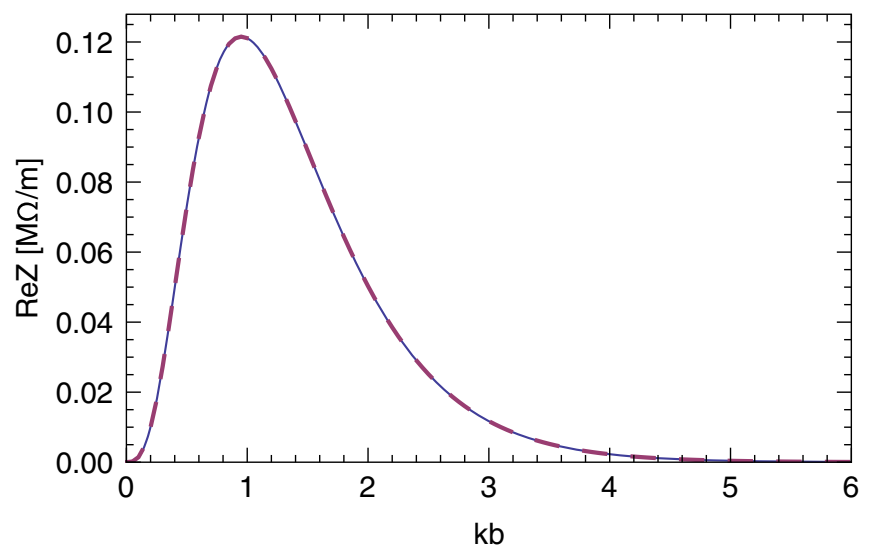

FIG. 6. $\operatorname{Re}(Z)$ for beam passing by one, infinitely wide dechirper plate at a distance $b=0.25 \mathrm{~mm}$ (blue). The analytical results, Eq. (29), are also shown (red dashes). 


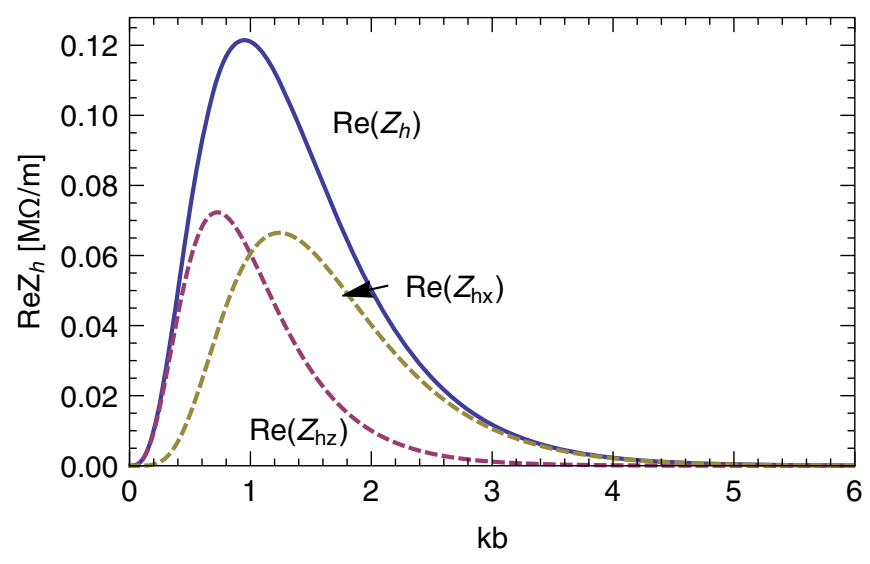

FIG. 7. The real part of the Joule heating impedance $\operatorname{Re}\left(Z_{h}\right)=$ $\operatorname{Re}\left(Z_{h z}\right)+\operatorname{Re}\left(Z_{h x}\right)$ for the beam passing by a single dechirper plate, for the case of plate width $w \rightarrow \infty$ (blue curve), with the constituent parts given in dashes. The beam passes by at offset $b=0.25 \mathrm{~mm}$ from the plate. The area under $\operatorname{Re}\left(Z_{h z}\right)\left[\operatorname{Re}\left(Z_{h x}\right)\right]$ is $42 \%$ [58\%] that under $\operatorname{Re}(Z)$.

becomes singular. However, the integral can be performed as a Cauchy integral, yielding a finite result (see the Appendix for details). The result is

$$
\operatorname{Re}(Z)=\left.\frac{2 \pi}{c} \frac{k \xi}{1+\xi} e^{-2 \xi b / h}\right|_{\xi=-1+\sqrt{1+k^{2} h^{2}}}
$$

where $h$ is the depth of corrugation. Note that the frequency at the peak can be approximated as $(k)_{\text {peak }}=\sqrt{3 /(2 h b)}$, which is similar to the perturbation peak frequency formula for the two plate case, $(k)_{\text {peak }}=\sqrt{2 /(h a)}$, with $a$ the half gap. The result of Eq, (29) is given by the red dashes in Fig. 6; we see that this function is almost identical to the earlier, numerical result that included wall resistance.

We next insert the single-plate magnetic fields, Eq. (27), into Eqs. (18) and (20) (but divided by 2, since there is only one plate) and numerically integrate to obtain the Joule heating impedance for the single, infinitely wide plate example. In Fig. 7 we show the real part of the Joule heating impedances $\operatorname{Re}\left(Z_{h z}\right), \operatorname{Re}\left(Z_{h x}\right)$ (the components with wall currents aligned in, respectively, the $z$ and $x$ directions) that we obtain. Again the beam is assumed to pass by at offset $b=0.25 \mathrm{~mm}$ from the plate. As expected, the total Joule heating impedance curve obtained numerically is the same, to good accuracy, as the impedance curve of Fig. 6. The area under $\operatorname{Re}\left(Z_{h z}\right)\left[\operatorname{Re}\left(Z_{h x}\right)\right]$ is $42 \%$ [58\%] that under $\operatorname{Re}(Z)$.

Finally, we insert the single-plate magnetic fields, Eq. (27), into Eqs. (17) and (19) (again divided by 2, since there is only one plate) and numerically integrate to find the Joule heating impedance for a beam passing by a single plate with finite width $w=12 \mathrm{~mm}$. The results are shown in Fig. 8. This time the area under the $\operatorname{Re}\left(Z_{h z}\right)$

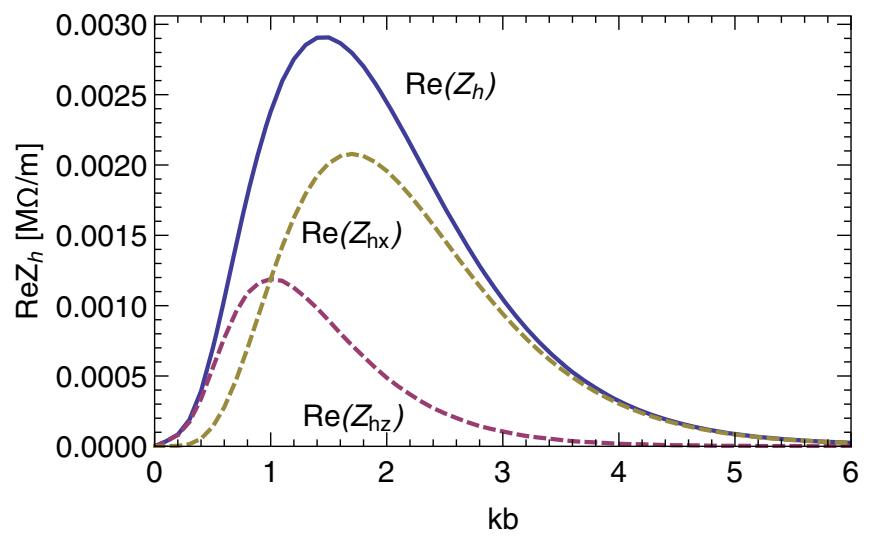

FIG. 8. The real part of the Joule heating impedance $\operatorname{Re}\left(Z_{h}\right)=$ $\operatorname{Re}\left(Z_{h z}\right)+\operatorname{Re}\left(Z_{h x}\right)$ for the beam passing by a single dechirper plate, for the case of plate width $w=12 \mathrm{~mm}$ (blue curve), with the constituent parts given in dashes. The beam passes by at offset $b=0.25 \mathrm{~mm}$ from the plate. The area under $\operatorname{Re}\left(Z_{h z}\right)\left[\operatorname{Re}\left(Z_{h x}\right)\right]$ is $0.9 \%$ [2.4\%] that under $\operatorname{Re}(Z)$, the beam impedance curve given in Fig. 6.

$\left[\operatorname{Re}\left(Z_{h x}\right)\right]$ curve is $0.9 \%$ [2.4\%] of that under the impedance curve $\operatorname{Re}(Z)$. Thus, $u_{h} / u_{w}=0.033$.

The short-range, point charge wake of a beam passing by a single plate of a flat dechirper at offset $b$ is [11]

$$
w_{z}(s)=\frac{1}{b^{2}} e^{-\sqrt{s / s_{0 l}}},
$$

with $\quad s_{0 l}=2 b^{2} t /\left(\pi \alpha^{2} p^{2}\right) \quad$ and $\quad \alpha=1-0.465 \sqrt{t / p}-$ $0.070(t / p)$. For corrugation parameters of Table I, $\alpha=0.636$. With distance from wall $b=250 \mu \mathrm{m}$, $s_{0 l}=98 \mu \mathrm{m}$. For the uniform bunch distribution of Table I, using Eq. (26), we find that $\varkappa=48 \mathrm{kV} /(\mathrm{pC} * \mathrm{~m})$. The loss of a point charge beam is $\varkappa(0)=1 /\left(2 b^{2}\right)$; thus, $\varkappa / \varkappa(0)=0.67$. The power lost by the beam is $P_{w}=Q^{2} \varkappa f_{\text {rep }}=434 \mathrm{~W} / \mathrm{m}$. Thus our analytical estimate of the Joule losses for the $12 \mathrm{~mm}$-wide dechirper plate is the fraction $\left(u_{h} / u_{w}\right)=0.033$ of this, or $P_{h}=14 \mathrm{~W} / \mathrm{m}$.

\section{NUMERICAL TIME-DOMAIN COMPARISONS}

The analytical model for the short-range wakes of an LCLS-type beam passing between two jaws of the RadiaBeam/LCLS dechirper has been verified in Ref. [10] using the finite difference, time-domain, wakefield solving program $\mathrm{ECHO}(2 \mathrm{D})$ [16]. However, in our analytical Joule heating calculations, for the case of finitewidth plates, we assumed that reflections from the sidewalls (in $x$ for a vertical dechirper) are negligible. To see how good this approximation is, we have performed numerical, time-domain calculations using the program CST PS, both for an example with the beam on axis between two dechirper plates, and for an example with the beam passing by a single plate. For the case of two dechirper plates, the accuracy of CST PS simulations 


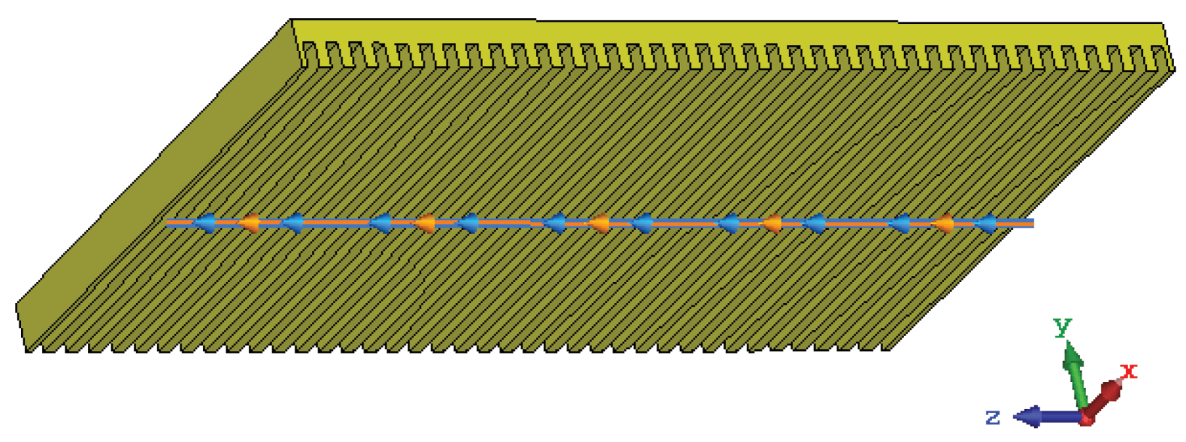

FIG. 9. Model used in the single-plate, numerical (CST STUDIO) calculations. The line and symbols indicate the beam trajectory. The plate width is $w=12 \mathrm{~mm}$, and, nominally, the beam offset is $b=0.25 \mathrm{~mm}$.

was verified by cross-checking with results using the wakefield code PBCI [14]. Since the bunches are short $\left(z_{r m s} \sim 20 \mu \mathrm{m}\right)$, the catch-up distance $\left[z_{c u} \sim a^{2} /\left(2 \sigma_{z}\right)\right]$ is large (on the order of cm's). However, the most challenging simulation issue is the long damping time of the fields that need to be followed for the Joule loss calculation. In the double plate case this time is on the order of cm's/c.

Figure 9 shows the geometry of the single corrugated plate and the (nominal) beam path used in the simulations. For modeling the lossy metal, a resistive wall impedance boundary condition for aluminum is applied. The corrugated plate structure is enclosed within a larger computational box that has free-space boundary conditions applied on all sides. This is necessary for modeling field radiation and thus, for the proper computation of the Joule losses. Such boundary conditions are provided by CST PS. This is why all Joule loss results in the following were obtained using this program. To confirm that the choice of bounding box does not affect the results, we performed one calculation with changed bounding box dimensions and found that the wake obtained was left unchanged.

The simulations are performed in the time domain by tracking a single bunch along the beam path until the wake potential and Joule loss per unit length saturate to steady state. Typical plate lengths considered in the simulations are some tens of cm's. To make the calculations manageable, for both double- and single-plate cases, we use Gaussian bunches with $\sigma_{z}=100 \mu \mathrm{m}$, i.e. significantly longer than our nominal bunch length. The resulting mesh with the necessary numerical resolution consists of up to $1.5 \times 10^{9}$ mesh points, and it typically takes several days to complete just one simulation. Since the typical bunch frequency is much higher than the structure frequency, the ratio $\left(u_{h} / u_{w}\right)$ will be about the same for this bunch length as for the target rms bunch length (see Table I), $\sigma_{z}=17 \mu \mathrm{m}$.

\section{A. Beam on axis between two dechirper plates}

For the two-plate case, the nominal half aperture is $a=0.7 \mathrm{~mm}$. The numerically obtained, steady-state bunch wake, for the Gaussian bunch, $\bar{w}_{z}(s)$ is given in Fig. 10. We see that the wake damps away on a scale of $s \sim 150 \mathrm{~mm}$. Fourier transforming the wake, we obtain the impedance (see Fig. 11). We find that $\operatorname{Re}(Z)$ is given by a collection of spikes (dominated by the first one) beginning with $k a=1.36,1.53,1.77$; note that these values agree with results of mode matching calculations, applied to the same geometry [8]. This behavior is quite different than our analytical, perturbative solution, with its one spike at $k a=1.67$ (see Fig. 3). This mismatch was expected.

For the numerical Joule loss calculations, the beam passed by a two-plate dechirper of length $L=135 \mathrm{~mm}$. From the magnetic fields at the plate surfaces, the Joule heating power at each time step was obtained. Figure 12 shows the results for the nominal $a=0.7 \mathrm{~mm}$ case (blue, solid curve); the steady state was obtained by extrapolation using a double exponential fitting function (the dashes). The final result is $P_{h}=13.5 \mathrm{~W} / \mathrm{m}$. The wake power loss for the $\sigma_{z}=100 \mu \mathrm{m}$ Gaussian bunch $P_{w}=108 \mathrm{~W} / \mathrm{m}$. Thus, the ratio of Joule heating and wake energy, according to the numerical calculation, is $\left(u_{h} / u_{w}\right)_{\text {num }}=P_{h} / P_{w}=0.125$, which is a factor of 4 larger than the analytical result obtained above,

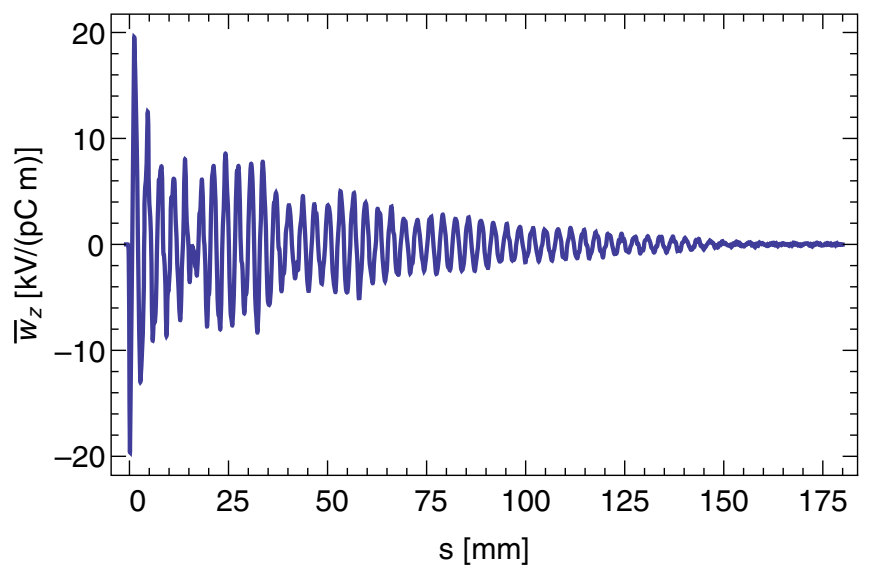

FIG. 10. The numerically obtained, longitudinal wake for the beam moving on axis of a double plate dechirper. Here half aperture $a=0.7 \mathrm{~mm}$ and dechirper length $L=135 \mathrm{~mm}$; the driving bunch is Gaussian with $\sigma_{z}=100 \mu \mathrm{m}$. Note that the wake is normalized to structure length. 


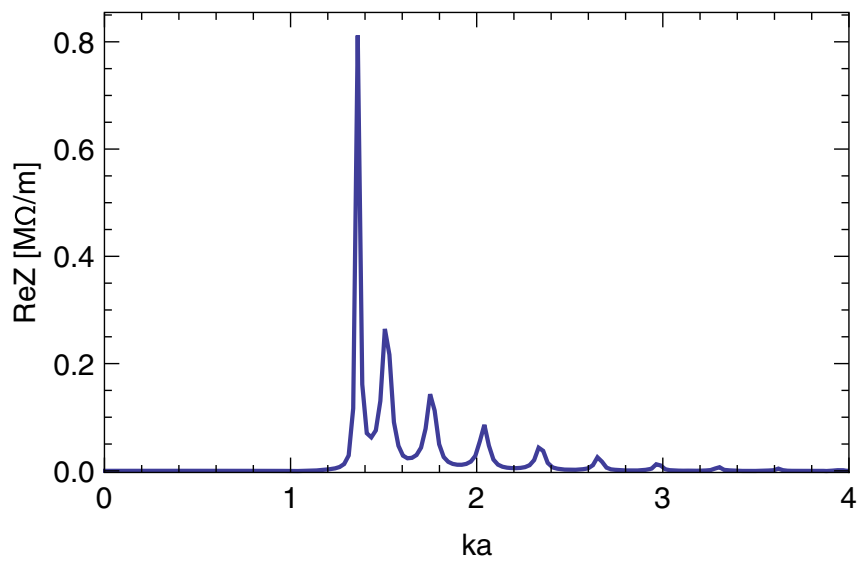

FIG. 11. The real part of the longitudinal impedance for the beam moving on axis of a double plate dechirper with half aperture $a=0.7 \mathrm{~mm}$, obtained by taking the Fourier transform of the numerically obtained wake of Fig. 10.

$\left(u_{h} / u_{w}\right)_{\text {ana }}=0.030$. Our best estimate for the Joule power loss for the LCLS-II beam, $\bar{P}_{h}$, is obtained taking $\left(u_{h} / u_{w}\right)_{\text {num }}$ and multiplying it with the wake power (analytically obtained above) for the short, uniform LCLS-II bunch shape, $\left(P_{w}\right)_{\text {ana }}$; i.e.

$$
\bar{P}_{h}=\left(u_{h} / u_{w}\right)_{\text {num }}\left(P_{w}\right)_{\text {ana }} .
$$

For the nominal case we obtain $\bar{P}_{h}=(0.125)(170 \mathrm{~W} / \mathrm{m})=$ $21.0 \mathrm{~W} / \mathrm{m}$.

As a final word on the two-plate calculation, note that a second case was also simulated, with $a=1.4 \mathrm{~mm}$. In this case, $(h / a)=0.35$ is half the size of before, and we find that the impedance (not shown) is closer to the analytical one. The simulation finds that the Joule power $P_{h}=2.64 \mathrm{~W} / \mathrm{m}$

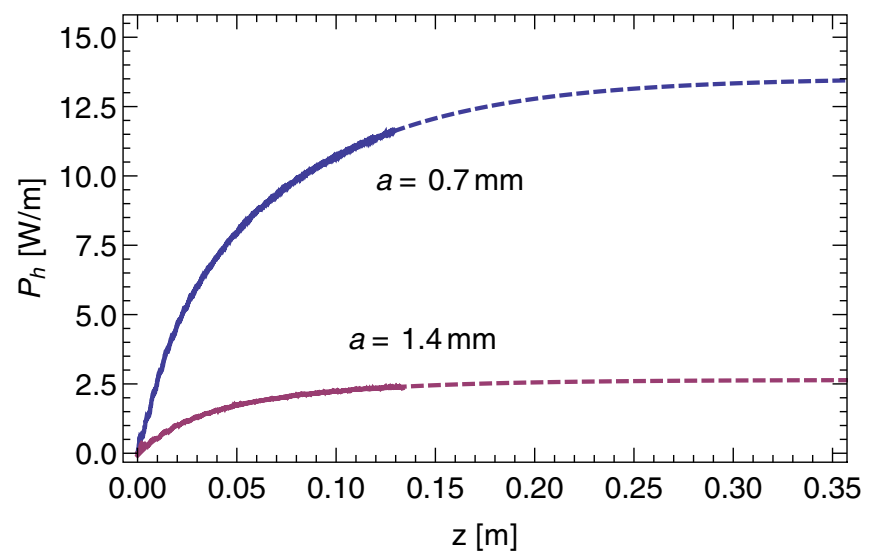

FIG. 12. Beam on axis between two plates: Joule power calculations obtained by time-domain simulation, for plate half aperture $a=0.7 \mathrm{~mm}$ (blue) and $a=1.4 \mathrm{~mm}$ (blue). The beam traverses the plate from its beginning, at $z=0$, to its end, at $z=0.135 \mathrm{~m}$. The dashed lines give extrapolation to steady state. Here $Q=300 \mathrm{pC}, f_{\text {rep }}=300 \mathrm{kHz}$; the driving charge is Gaussian with $\sigma_{z}=100 \mu \mathrm{m}$.

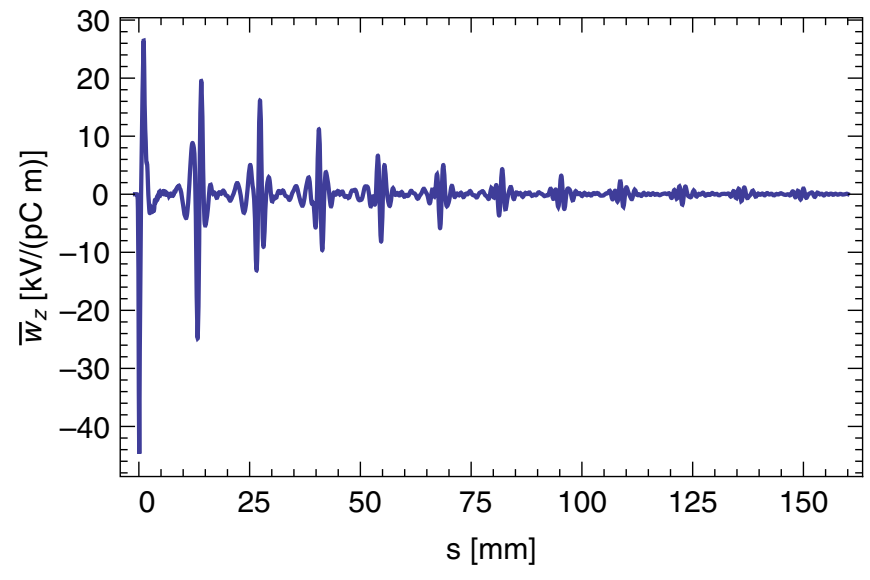

FIG. 13. The numerically obtained, longitudinal wake for a beam moving past a single-plate dechirper. Here the beam offset $b=0.25 \mathrm{~mm}$ and dechirper length $L=115 \mathrm{~mm}$; the driving bunch is Gaussian with $\sigma_{z}=100 \mu \mathrm{m}$. Note that the wake is normalized to structure length.

(see Fig. 12, the red curve). The energy ratio $\left(u_{h} / u_{w}\right)_{\text {num }}=$ 0.065 , which is a factor of 2.5 larger than the analytical value $\left(u_{h} / u_{w}\right)_{\text {ana }}=0.025$, rather than the factor of 4 we had before. For $a=1.4 \mathrm{~mm},\left(P_{w}\right)_{\text {ana }}=46 \mathrm{~W} / \mathrm{m}$, and our best estimate of Joule power loss becomes $\bar{P}_{h}=(0.065)(46 \mathrm{~W} / \mathrm{m})=3.0 \mathrm{~W} / \mathrm{m}$.

\section{B. Beam passing by a single corrugated plate}

The numerically obtained wake for a $100 \mu \mathrm{m}$ Gaussian bunch passing at distance $b=0.25 \mathrm{~mm}$ from the plate is shown in Fig. 13. Here we see that the wake dies out after $s \sim 120 \mathrm{~mm}$ and that there are reflections from the sides of the plate. Note that since the period in the reflections is $\sim 13.5 \mathrm{~mm}$ (and not equal to the plate width, $w=12 \mathrm{~mm}$ ), we infer that the average group velocity of the waves moving sideways is $v_{g}=(12 / 13.5) c=0.89 c$.

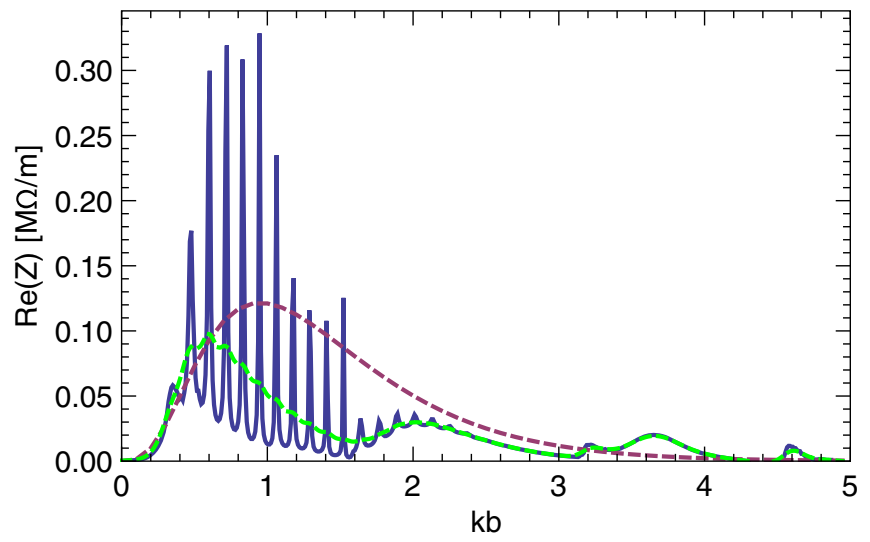

FIG. 14. The real value of the impedance $\operatorname{Re}(Z)$ for the singleplate example (blue curve). The broadband impedance obtained from the same wake is given by green dashes. The analytical perturbation result (Fig. 6) is given by red dashes. 


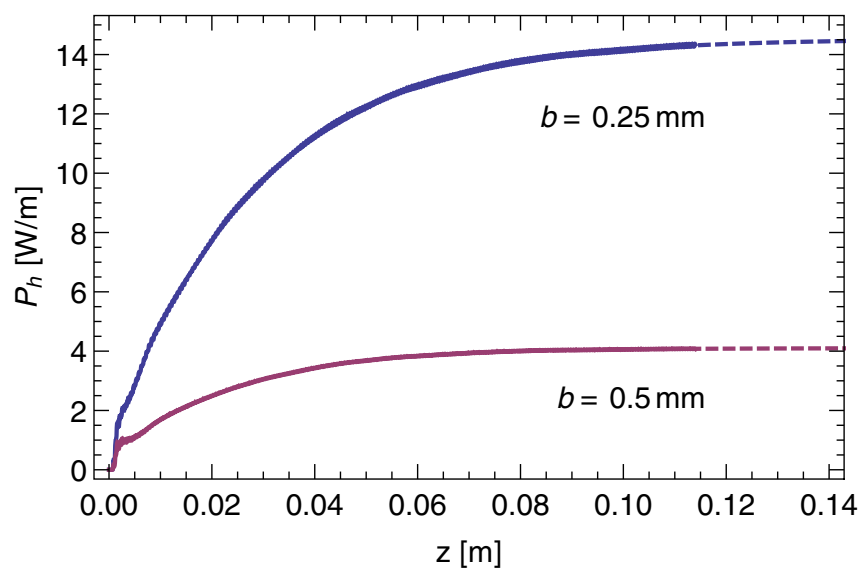

FIG. 15. Single plate: numerically obtained, Joule power calculations for beam offsets $b=0.25 \mathrm{~mm}$ (blue) and $b=$ $0.5 \mathrm{~mm}$ (red). The beam traverses the plate from its beginning, at $z=0$, to its end, at $z=0.115 \mathrm{~m}$. The dashed lines give extrapolation to steady state. Here $Q=300 \mathrm{pC}, f_{\text {rep }}=300 \mathrm{kHz}$; the driving charge is Gaussian with $\sigma_{z}=100 \mu \mathrm{m}$.

The real part of the impedance $\operatorname{Re}(Z)$ for the single-plate example is given in Fig. 14 (blue curve). To obtain this, the longitudinal wake (Fig. 13) was Fourier transformed and multiplied by $e^{k^{2} \sigma_{z}^{2} / 2}$. The narrow, evenly spaced spikes in the impedance [with spacing $\Delta f \approx c /(13.5 \mathrm{~cm})$ ] are due to the reflections in the wake. A short bunch, however, cannot resolve these spikes. To generate a "broadband impedance," one that is easier to compare with our analytical result, we multiplied the wake by a Gaussian form factor, with rms length $\sigma=5 \mathrm{~mm}$, before Fourier transforming. The resulting impedance is given by green dashes in the figure. Our analytical result (Fig. 6) is given in red dashes. Although the low and higher frequency behaviors of the red and green curves agree well, the numerical peak is narrower and the frequency of the peak is lower than the analytical one. This disagreement appears to be a consequence of the corrugation parameters not being in the perturbative regime: here $(h / b)=2$, which is not small compared to 1 .

For Joule loss simulations, the beam was passed by a single-plate dechirper of length $L=115 \mathrm{~mm}$. Figure 15 shows the results for the nominal $b=0.25 \mathrm{~mm}$ case (blue, solid curve), and the extrapolation to steady state (the dashes). The steady-state result is $P_{h}=14.5 \mathrm{~W} / \mathrm{m}$.
The wake power loss for the $\sigma_{z}=100 \mu \mathrm{m}$ Gaussian bunch $P_{w}=264 \mathrm{~W} / \mathrm{m}$. Thus, the ratio of Joule heating and wake energy according to the numerical calculation is $\left(u_{h} / u_{w}\right)_{\text {num }}=P_{h} / P_{w}=0.055$; this is a factor of 1.67 larger than the analytical result obtained above, $\left(u_{h} / u_{w}\right)_{\text {ana }}=0.033$. This is due to the multiple reflections of the wakefield from the sides of the plate that are not considered in the analytical model. Our best estimate of the Joule power loss for the LCLS-II beam, $\bar{P}_{h}=$ $\left(P_{w}\right)_{\text {ana }}\left(u_{h} / u_{w}\right)_{\text {num }}=24.0 \mathrm{~W} / \mathrm{m}$.

More single-plate simulations were performed for larger beam offsets: $b=0.5,1.0,1.5 \mathrm{~mm}$. As we move to ever smaller values of $(h / b)$, the numerically obtained (broadband) impedance (not shown) agrees better with the analytical one. The numerical energy ratio $\left(u_{h} / u_{w}\right)$, however, remains about a factor of 2 larger than the analytical one. For $b=0.5 \mathrm{~mm}$, the simulations find that the Joule power $P_{h}=4.0 \mathrm{~W} / \mathrm{m}$ (see Fig. 15 , the red curves). The energy ratio $\left(u_{h} / u_{w}\right)_{\text {num }}=0.035$, and our best estimate of Joule power loss becomes $\bar{P}_{h}=4.6 \mathrm{~W} / \mathrm{m}$. Finally, note that our nominal, two-plate and single-plate results are summarized in Table II.

\section{CONCLUSIONS}

We have performed Joule power loss calculations for the new RadiaBeam/LCLS-II dechirper, whose engineering details-for example concerning the cooling requiredare still being finalized. We have investigated the configurations of the beam on axis between the two plates, for chirp control, and for the beam especially close to one plate, for use as a fast kicker. Our calculations involve an analytical model that uses a surface impedance approach, valid for perturbatively small dechirper parameters. In addition, our model ignores effects of field reflections at the sides of the dechirper plates, and is thus expected to underestimate the Joule losses. The analytical results were also tested by numerical, time-domain simulations using computer programs in CST STUDIO and PBCI. We find that most of the wake power lost by the beam is radiated out to the sides of the plates. While our theory can be applied to the LCLS-II dechirper with large gaps, for the nominal apertures we are not in the perturbative regime and the reflection contribution to Joule losses is not negligible. With input from computer simulations, we estimate the

TABLE II. Summary of Joule heating calculations for the LCLS-II dechirper, giving case; wake power lost by beam, $\left(P_{w}\right)_{\text {ana }}$; ratio of energy in Joule heating and beam energy loss, analytical calculation, $\left(u_{h} / u_{w}\right)_{\text {ana }}$; Joule power loss, analytical calculation, $\left(P_{h}\right)_{\text {ana }}$; energy ratio, according to numerical calculation, $\left(u_{h} / u_{w}\right)_{\text {num }}$; and our best estimate of Joule losses, $\bar{P}_{h}=\left(u_{h} / u_{w}\right)_{\text {num }}\left(P_{w}\right)_{\text {ana. }}$. Both cases assume the high charge scenario, with $Q=$ $300 \mathrm{pC}$ and $f_{\text {rep }}=100 \mathrm{kHz}$; the bunch shape is taken as uniform, with total length $\ell=60 \mu \mathrm{m}$.

\begin{tabular}{lccccc}
\hline \hline Case & $\left(P_{w}\right)_{\text {ana }}[\mathrm{W} / \mathrm{m}]$ & $\left(u_{h} / u_{w}\right)_{\text {ana }}$ & $\left(P_{h}\right)_{\text {ana }}[\mathrm{W} / \mathrm{m}]$ & $\left(u_{h} / u_{w}\right)_{\text {num }}$ & $\bar{P}_{h}[\mathrm{~W} / \mathrm{m}]$ \\
\hline Two plates, $a=0.7 \mathrm{~mm}$ & 170 & 0.030 & 5 & 0.125 & 21.0 \\
Single plate, $b=0.25 \mathrm{~mm}$ & 435 & 0.033 & 14 & 0.055 & 24.0 \\
\hline \hline
\end{tabular}


Joule power loss (assuming bunch charge of $300 \mathrm{pC}$, repetition rate of $100 \mathrm{kHz}$ ) is $21 \mathrm{~W} / \mathrm{m}$ for the case of two plates, and $24 \mathrm{~W} / \mathrm{m}$ for the case of a single plate.

The single-plate configuration of a dechirper has, until now, received little attention in the literature. In this report we have presented also the impedance of a beam passing by a single corrugated plate.

\section{ACKNOWLEDGMENTS}

We thank I. Zagorodnov for helpful discussions on the subject of the short-range wakefields in the dechirper. This work was supported by the U.S. Department of Energy, Office of Science, Office of Basic Energy Sciences, under Contract No. DE-AC02-76SF00515.

\section{APPENDIX: DERIVATION OF $\operatorname{Re}(Z)$ FOR BEAM PASSING BY A SINGLE DECHIRPER PLATE}

The impedance is

$$
Z(\omega)=-\frac{1}{2 \pi Q} \int_{-\infty}^{\infty} d q \hat{E}_{z}(q, 0, \omega)
$$

where [see Eq. (28)]

$\hat{E}_{z}(q, 0, \omega)=-\frac{4 \pi Q}{c} \frac{\zeta_{z} k|q| e^{-2 b|q|}}{k|q|\left(1+\zeta_{z} \zeta_{x}\right)+i \zeta_{z}\left(q^{2}-k^{2}\right)}$.

We need to calculate the following integral:

$Z(\omega)=\frac{4}{c} \zeta_{z} k \int_{0}^{\infty} d q \frac{q e^{-2 b q}}{k q\left(1+\zeta_{z} \zeta_{x}\right)+i \zeta_{z}\left(q^{2}-k^{2}\right)}$

(where we have used the fact that the integrand is symmetric with respect to $q$ ). In the limit when the resistive term $\zeta_{r w} \rightarrow 0$, we have $\zeta_{x}=0$ and $\zeta_{z}=-\frac{1}{2} i h k$ and the integral reduces to

$$
Z(\omega)=-\frac{4 i}{c} h k^{2} \int_{0}^{\infty} d q \frac{q e^{-2 b q}}{2 k q+h k\left(q^{2}-k^{2}\right)} .
$$

It is easy to see that the denominator of the integrand vanishes at

$$
q=q_{r}=\frac{-1+\sqrt{1+h^{2} k^{2}}}{h},
$$

and the integrand has a pole at this point. This pole has to be bypassed in the complex plane (of variable $q$ ) or, equivalently, the integration path needs to be shifted from the real axis. The direction of the shift can be found by analyzing the position of the pole when $\zeta_{r w}$ is small, but not equal to zero. This analysis shows that for nonzero $\zeta_{r w}$ the pole has a positive imaginary part, which means that in the limit

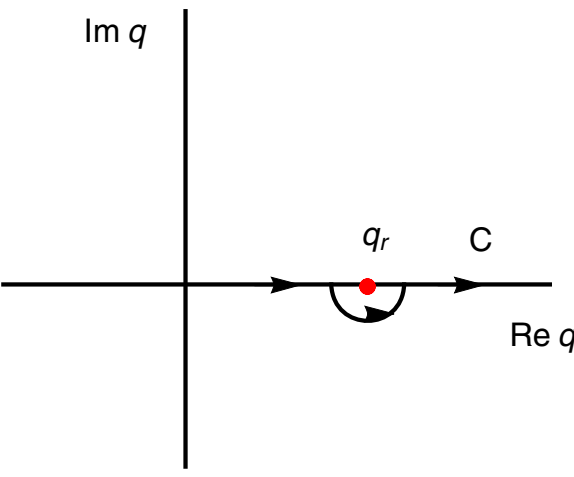

FIG. 16. Integration path $C$ in the complex plane of variable $q$.

$\zeta_{r w} \rightarrow 0$ the integration path should be modified as shown in Fig. 16.

We are interested in calculating the real part of the impedance, $\operatorname{Re}(Z)$. Because of the imaginary factor in front of the integral (A4), the real part of $Z$ is equal to the half residue of the integrand at $q=q_{r}$. A straightforward calculation of the residue yields

$$
\operatorname{Re}(Z)=\left.\frac{2 \pi}{c} \frac{k \xi}{1+\xi} e^{-2 \xi b / h}\right|_{\xi=-1+\sqrt{1+k^{2} h^{2}}} .
$$

[1] K. Bane and G. Stupakov, Corrugated pipe as a beam dechirper, Nucl. Instrum. Methods Phys. Res., Sect. A 690, 106 (2012).

[2] M. Guetg et al., Commissioning of the RadiaBeam/SLAC dechirper, in Proceedings of the 7th International Particle Accelerator Conference (IPAC 2016), Busan, Korea, 2016 (CERN, Geneva, 2016), p. 809.

[3] A. Lutman et al., Fresh-slice multicolor x-ray free-electron lasers, Nat. Photonics 10, 745 (2016).

[4] K. Bane and G. Stupakov, Surface impedance formalism for a metallic beam pipe with small corrugations, Phys. Rev. ST Accel. Beams 15, 124401 (2012).

[5] K. Bane and G. Stupakov, Using surface impedance for calculating wakefields in flat geometry, Phys. Rev. ST Accel. Beams 18, 034401 (2015).

[6] K. Bane and G. Stupakov, Erratum: Using surface impedance for calculating wakefields in flat geometry, Phys. Rev. Accel. Beams 19, 039901(E) (2016).

[7] K. Bane and G. Stupakov, Impedance of a rectangular beam tube with small corrugations, Phys. Rev. ST Accel. Beams 6, 024401 (2003).

[8] Z. Zhang, K. Bane, Y. Ding, Z. Huang, R. Iverson, T. Maxwell, G. Stupakov, and L. Wang, Electrobeam energy chirp control with a rectangular corrugated structure at the Linac Coherent Light Source, Phys. Rev. ST Accel. Beams 18, 010702 (2015).

[9] A. Novokhatski, Wakefield potentials of corrugated structures, Phys. Rev. ST Accel. Beams 18, 104402 (2015). 
[10] K. Bane, G. Stupakov, and I. Zagorodnov, Analytical formulas for short bunch wakes in a flat dechirper, Phys. Rev. Accel. Beams 19, 084401 (2016).

[11] K. Bane, G. Stupakov, and I. Zagorodnov, Wakefields of a beam near a single plate in a flat dechirper, Report No. SLAC-PUB-16881, 2016.

[12] K. Bane, G. Stupakov, and E. Gjonaj, Estimate of Joule heating in a flat dechirper, Report No. SLAC-PUB-16922, 2017.

[13] CST-Computer Simulation Technology, CST PARTICLE STUDIO, http://www.cst.com.
[14] E. Gjonaj, T. Lau, S. Schnepp, F. Wolfheimer, and T. Weiland, Accurate modeling of charged particle beams in linear accelerators, New J. Phys. 8, 285 (2006).

[15] A. Chao, Physics of Collective Beam Instabilities in High Energy Accelerators (John Wiley \& Sons, New York, 1993), Chap. 2.

[16] I. Zagorodnov, K. Bane, and G. Stupakov, Calculation of wakefields in 2D rectangular structures, Phys. Rev. ST Accel. Beams 18, 104401 (2015). 